\title{
Thermal regimes of space composite structures. Part II
}

\author{
Sergey Reznik ${ }^{1, *}$ \\ ${ }^{1}$ Bauman Moscow State Technical University, 105005 Moscow, Russia
}

\begin{abstract}
Composite materials have a unique combination of mechanical and thermal characteristics. This allows you to create lightweight, durable, heat-resistant design. In the article the common features for all space composite structures were highlighted. The article deals with the thermophysical problems arising in the design of space composite structures associated with strict requirements for their weight and functional efficiency. The feasibility of using combined heat transfer models to improve design accuracy has been shown.
\end{abstract}

\section{Space antenna reflectors}

\subsection{Evolution of onboard space antennas}

The first satellites were equipped with non-directional rod antennas, so to receive their weak signals large receiving ground antennas were required. Exploration of the solar system (first Venus and Mars, then Jupiter, Saturn, Uranus, Neptune and Mercury), as well as the development of the high-apogee elliptical orbits and geostationary orbit (GEO) called for devices to concentrate the radio waves - reflectors. As a rule, reflectors are open, gently sloped parabolic shells. Today reflectors can be fixed-shape, adaptive, shape-shifting within a predetermined range and transformable, with the antenna aperture diameter being much greater than that of the launch vehicle fairing (typically over 4 meters). Reflectors operate in $\mathrm{L}, \mathrm{Ka}, \mathrm{Ku}, \mathrm{V}$, and Q bands in 1-70 GHz range for the stationary and mobile "land - board of the satellite - land" radio communication, as well as the inter-satellite communication.

In space reflectors are unevenly heated by the heat radiation from the Sun and the Earth, which causes thermal deformations and shape deviation. To enhance the shape stability, a three-layer layout is most commonly used, where two thin composite shells are joined together by honeycombs of metal foil. Frames on the convex surface are used to increase the structural stiffness and to fix the reflector to the spacecraft. The areal density of the threelayer reflectors is $3.5-5.0 \mathrm{~kg} / \mathrm{m}^{2}$. A different layout, where ribs are positioned on the convex surface, enables stiff structures with a still lower areal density. Modern communication satellites are often equipped with several reflectors, so a lower areal density can noticeably decrease the structure weight. However, the choice of the ribs arrangement, shape, height and thickness requires further research.

\footnotetext{
*Corresponding author: sreznik@,bmstu.ru
} 


\subsection{Modern approaches to the design of space antenna reflectors}

Design requirements for the advanced space antenna reflector: accuracy of reflector surface (root-mean-square error less than $\Lambda / 16, \Lambda$ is wavelength and displacement $0.1 \mathrm{~mm}$ ); strength and stability during spacecraft launch; high dynamic characteristics (high frequencies of structures, not less than $100 \mathrm{~Hz}$ ); areal mass of the reflector assembly not greater than 2 $\mathrm{kg} / \mathrm{m}^{2}$; more than 15 years' operating cycle in space.

The aim of the work is to select the design layout of ultra-lightweight space antenna reflector from CFRP with high shape and size stability for advanced communication systems.

Why are CFRP best suited for antenna reflectors? Because CFRP have: high strength (unidirectional material); 1.3-2.1 GPa ultimate tensile strength; high stiffness (unidirectional material): 280-400 GPa tensile modulus; low temperature coefficient of linear expansion of $\pm 0.5 \cdot 10^{-6} \mathrm{~K}^{-1}$; thermal conductivity, within $\lambda_{\perp}=0,4-0,8 \mathrm{~W} /(\mathrm{m} \cdot \mathrm{K})$ range $; \lambda_{I I}=8-70 \mathrm{~W} /(\mathrm{m} \cdot \mathrm{K})$ $\left(300 \mathrm{~W} /(\mathrm{m} \cdot \mathrm{K})\right.$ for pitch-based carbon fibers); relatively small density $\left(1500-1650 \mathrm{~kg} / \mathrm{m}^{3}\right)$; moderate cost ( $\$ 1000$ per $\mathrm{kg}$ ); variety of manufacturing methods (infusion, hand layup, winding, etc.).

Many modern communication satellites are located in the geostationary orbit. At the same time, the spacecraft is constantly above one very large section of the earth surface, since it moves with the same angular velocity as Earth. In the geostationary orbit, spacecraft and its antennas are constantly illuminated by the direct solar heat flux for most part of the year. Usually, calculations are based on the average value of this flux, the so-called solar constant of $1368 \mathrm{~W} / \mathrm{m}^{2}$. Twice a year, during the 45 days of the spring and autumn equinox, the spacecraft is in the Earth's shadow. The maximum time spent in the shadow is about 71 minutes, where the spacecraft is exposed to the Earth own radiation, about $5.5 \mathrm{~W} / \mathrm{m}^{2}$. The experience of estimating the thermal regimes in GEO shows that even such weak thermal effects should not be neglected, otherwise the spacecraft temperature values are calculated erroneously low, especially in the case of thin-walled structures prone to rapid cooling due to low heat capacity. Because of these operating conditions, the thermal regimes for space antenna reflectors are estimated both the illuminated and for the shadow areas of the orbit.

The design process includes the following steps: selecting one design layout; defining the thermal loads on the reflector in GEO; creating the geometric and finite element model of the reflector; FE modelling of the thermal state using "Siemens space system analysis"; selecting the method of joining the reflector to the spacecraft; FE modeling of the stress-strain state using "Siemens NX Nastran"; determining the maximum thermal deformation and displacement of the reflecting surface; analyzing the results and comparing them with other design layout options.

\subsection{A hard-to-achieve combination of lightweight and stiffness}

The authors and their colleagues have presented the design studies of reflector structures made of carbon fiber with various ribs arrangements on the convex surface of the parabolic shell [1-4]. This paper, by way of example, presents the mathematical modeling results of thermal regimes for different design variants of a reflector: isogrid, five-pointed star and sixpointed star ribbed reinforcement arrangement (Table 1, 2, Fig. 1). In all cases, the reflectors were constructed of CFPR and had six layers made of the flattened plain weave ASPRO fabric and epoxy binder ED-20 with a thickness of 0.4-1.2 $\mathrm{mm}$. The plies were positioned at $\pm 90^{\circ} / \pm 90^{\circ} / \pm 90^{\circ}$. 
Table 1. Variants of ribbed reflectors: five-point star and six-point star arrangement.

\begin{tabular}{|l|c|c|c|c|}
\hline Name of variant & Ribs arrangement & $\begin{array}{c}\text { Height of } \\
\text { ribs, mm }\end{array}$ & $\begin{array}{c}\text { Thickness of } \\
\text { ribs, mm }\end{array}$ & $\begin{array}{c}\text { Thickness of } \\
\text { shell, mm }\end{array}$ \\
\hline Star6_25_12_06 & Six pointed star & 25 & 1.2 & 0.6 \\
\hline Star6_60_06_06 & Six pointed star & 60 & 0.6 & 0.6 \\
\hline Star6_90_06_04 & Six pointed star & 90 & 0.6 & 0.4 \\
\hline Star5_90_06_04 & Five pointed star & 90 & 0.6 & 0.4 \\
\hline Star5_53_06_06 & Five pointed star & 53 & 0.6 & 0.6 \\
\hline
\end{tabular}

Computer modelling demonstrated that the six-pointed star ribbed arrangement possessed superior weight characteristics and high dimension stability (Table 2).

Table 2. Comparison of ribbed design layouts.

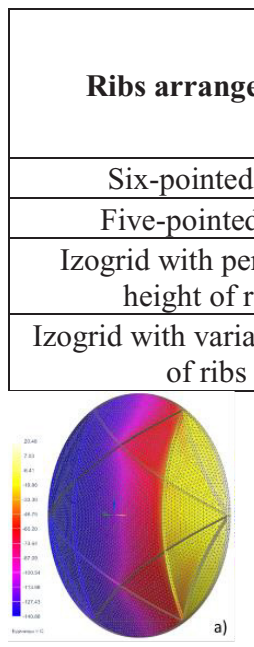

(a)

\begin{tabular}{l|r} 
ement & $\begin{array}{r}\text { Shell } \\
\text { ribs } \\
\text { thick } \\
\text { m }\end{array}$ \\
\hline star & 0.6 \\
\hline rabs star & 0.6 \\
\hline
\end{tabular}

(b)

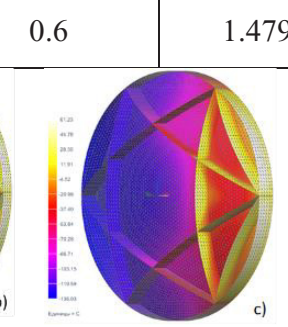

(c)

\begin{tabular}{|c|}
$\begin{array}{c}\text { Mass of the } \\
\text { reflector, } \\
\mathbf{~ k g}\end{array}$ \\
\hline 1.523 \\
\hline 1.502 \\
\hline 1.549 \\
\hline 1.479 \\
\hline
\end{tabular}

\begin{tabular}{|c|c|}
$\begin{array}{c}\text { Maximum } \\
\text { displacement of } \\
\text { reflecting } \\
\text { surface, } \mathbf{~ m m}\end{array}$ & $\begin{array}{c}\text { Linear } \\
\text { density, } \\
\mathbf{2} \\
\mathbf{~ k g / m}\end{array}$ \\
\hline 0.14 & 1.354 \\
\hline 0.12 & 1.328 \\
\hline 0.185 & 1.369 \\
\hline 0.18 & 1.308 \\
\hline & \\
\hline & \\
\hline & \\
\hline
\end{tabular}

(d) (e)

Fig. 1. Temperature state (degrees Celsius) for: a - "Star6 25 12 06", b - "Star6_60_06_06", c "Star6_90_06_04", d - "Star5_53_06_06", e - "Star5_90_06_04" with $270^{\circ}$ angle between reflector rotation axis and the Sun - Earth line.

\section{Reusable space vehicles}

\subsection{Thermal barrier on the way to reusable spacecraft and rockets}

Space vehicles were disposable only for the first 15 years after the First Sputnik had been launched. First-generation launch vehicles were modernized guided ballistic missiles designed to fly in one direction with small coefficients of safety. In 1972, the United States began creating the Space Shuttles, and the USSR in 1976 began working on a similar "Buran" - "Energy" system. Both types of reusable space vehicles (RSV) were designed for 100 flights to the low Earth orbit. The problem of thermal protection (TP) was one of the most difficult because with the RSV surface area of about $1000 \mathrm{~m}^{2}$, the use of traditional polymer $\mathrm{CM}$ was unacceptable for weight reasons. The way out was to utilize combinations of heatshielding $\mathrm{C}-\mathrm{C}$ elements, ceramic tiles, flexible coatings from heat-resistant fabrics [5]. After 30 years of the Space Shuttle operation, some of the most significant drawbacks were the extremely high labor costs associated with preflight preparation of the tiled TP, reaching about 40,000 man-hours. 
New generation of RSV required elements that could be replaced quickly and had larger dimensions than tens of thousands of 150x150 mm and 200x200 mm tiles in the first shuttles $[6,7]$. At the same time, more lightweight TP-materials were investigated consisting of sintered heat-resistant materials or lightweight wool-like (felt) alumina materials inside metal or composite containers or combinations with silica glass fibers with less than $100 \mathrm{~kg} / \mathrm{m}^{3}$ density. These materials comprise randomly oriented thin fibers (about $2 \mu \mathrm{m}$ in diameter) with a boron oxide binder. Porosity of the material exceeds $94 \%$. The heat transfer process in such materials is combined. The front surface of the TP element has several functions. It prevents erosion of fibers by high-speed air flows and precipitation, has low catalytic activity and high emissivity, which reduces the heat flux to the fibrous insulation. Nevertheless, as a result of aerodynamic heating, this surface temperature reaches more than $1500 \mathrm{~K}$ with the excess heat is radiated into the outer space and into the interior of the porous material, where it is absorbed and scattered. Such a complex picture of heat transfer in a highly porous material is crucial for determining its effective coefficient of thermal conductivity, which is used in designing of the TP.

\subsection{Heat transfer in high-porosity materials for thermal protective system}

The TP of RSV requires technically feasible and inexpensive materials with low density, low heat conductivity, high thermal stability. The create TP with optimum weight-geometric and economic characteristics it is of high significance to determine the main performance characteristics of porous materials under operating conditions. However, these data for the vast majority of materials, especially the coefficient of thermal conductivity, only exist in a limited temperature range or do not exist at all. At the same time, with the current laboratory equipment we can measure only the effective coefficient of thermal conductivity of semitransparent materials, characterizing the total heat transfer, whose values depend strongly on the measuring conditions.

In terms of thermal design, it is extremely challenging to rationally choose the mathematical model of heat exchange in a layer of semi-transparent scattering material, such as high porosity ceramics. The problem is in the close interconnection of the temperature and radiation fields in these materials which means solving essentially nonlinear problems of radiative-conductive heat transfer, that include the heat conduction and radiation transfer equations. Solving the transfer equation directly, for example using the discrete ordinates method, will lead to excessive computational burden, which unsuitable at the thermal design stage. The previous research [8] showed that the necessary balance of performance and accuracy is achieved when using the diffusion approximation for the radiation transfer equation. In the future, when computer technology is better developed (multi-core and multiprocessor systems, GPGPU), it may be reasonable to use a more accurate method for solving the radiation transfer equation: the half moment method.

The aim of the work is to investigate the combined heat transfer regularities in porous TP materials for RSV at wide temperatures interval $(300-2000 \mathrm{~K})$ and at high rates of heating (up to $100 \mathrm{~K} / \mathrm{s}$ ).

\subsection{Methodology of theoretical and experimental investigation}

The main approaches to this problem are: mathematical simulation and design of thermal test conditions; thermal tests of new porous materials samples and measurements of temperature distribution inside the samples; heating methods - contact, radiative or convective; solution of a 1-d and 2-d inverse problems of effective thermal conductivity (IP ETC) and radiative and conductive heat transfer (IP RCHT). 
Modern methods for solving IP RCHT are based on the comparison of calculated and experimentally measured temperature values in a sample of semi-transparent material. Obviously, IP ETCs are a special case of IP RCHT. Probably, the pioneering work in this direction is [8], where the problem of determining the temperature dependence of the thermal conductivity in a semi-transparent scattering material was solved for the first time using experimental data corresponding to nonstationary heat transfer in the Buran TPC. Later this approach developed into more complex multidimensional problems $[9,10]$ and methods for determining the coefficient of semi-transparent materials thermal conductivity adapted for processing the investigation results using laboratory facilities [11-13].

Our theoretical and experimental investigations have proved that for highly porous semitransparent materials, the effective thermal conductivity coefficient depends most significantly on the rate and period of heating. It has been established that for the optical layer thickness exceeding 100, the effective thermal conductivity coefficient at $3 \mathrm{~K} / \mathrm{s}$ surface heating rate (curve 3 in Fig. 2) practically coincides with the value of the effective thermal conductivity coefficient obtained in the Rosseland approximation (curve 2 in Fig. 1), and at the surface heating rate exceeding $300 \mathrm{~K} / \mathrm{s}$ it is close to the molecular coefficient of thermal conductivity (curves 1 and 5 in Fig. 2). At the heating rate of more than $3 \mathrm{~K} /$ and less than $300 \mathrm{~K} / \mathrm{s}$, the effective coefficient of thermal conductivity lies between the values of the effective thermal conductivity coefficient in the Rosseland approximation and the molecular coefficient of thermal conductivity, tending to the latter as the heating rate increases (curves 4 and 5 in Fig. 2) [14].

The study of the temperature dependencies of the effective thermal conductivity coefficient of heat-insulating materials with varying densities [15] has shown that at the temperature of up to $600 \mathrm{~K}$, when the heat transfer is mainly conducted along a solid framework and a gaseous medium, the material with the lowest density has the lowest effective heat conductivity (curve 1 in Fig. 3). However, in the region of high temperatures, when the share of radiative heat transfer to the total heat transfer increases, the effective thermal conductivity is lower for denser materials (curve 3 in Fig. 3). This is explained by the fact that as the density of the material increases, the concentration of fibers per unit volume increases, which causes an increase in the scattering coefficient and, as a consequence, a decrease in the radiative energy transfer. This result opens up possibilities for optimizing the constructions of TP multilayered elements using heat-insulating materials of variable density depending on the specific conditions of thermal loading (Fig. 3).

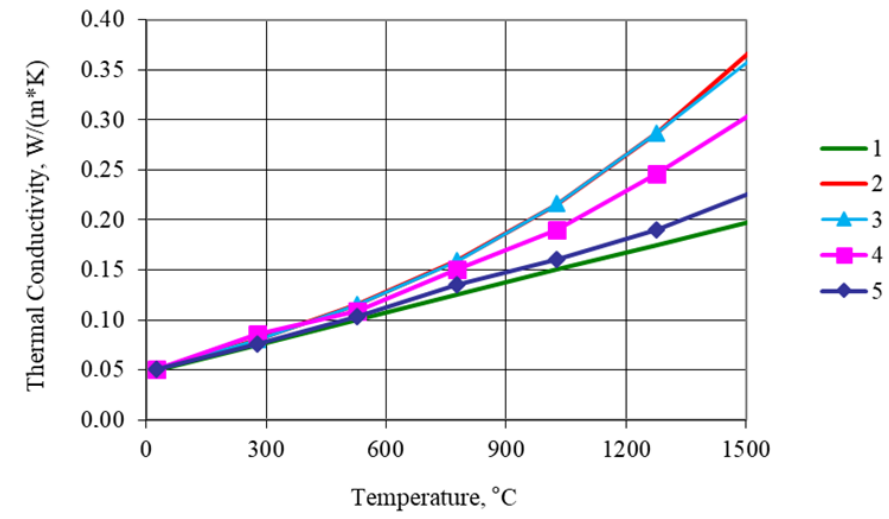

Fig. 2. Temperature dependences of the thermal conductivity of a semi-transparent scattering material: 1 - conductive coefficient of thermal conductivity; 2 - effective coefficient of thermal conductivity (Rosseland); 3 - effective coefficient of thermal conductivity at $3 \mathrm{~K} / \mathrm{s}$ rate of heating of the frontal surface; 4 - the same for $30 \mathrm{~K} / \mathrm{s} ; 5$ - the same for $300 \mathrm{~K} / \mathrm{s}$. 


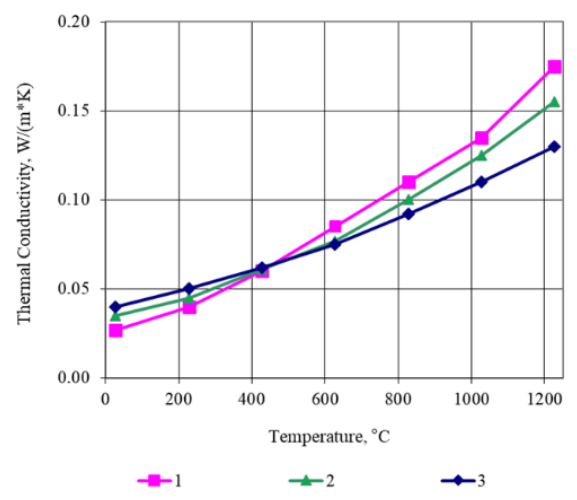

Fig. 3. Temperature dependencies of the ETC coefficient of silica fibrous materials: 1 - with 90 $\mathrm{kg} / \mathrm{m}^{3}$ density; $2-105 \mathrm{~kg} / \mathrm{m}^{3}$ density; $3-120 \mathrm{~kg} / \mathrm{m}^{3}$ density.

\section{Conclusion}

The increasingly complex and ambitious challenges of space exploration stimulate composite structures development. Experience shows that the design layouts of any spacecraft must be selected in view of the expected thermal regimes. Even though there is an ample variety of space composite structures, there exist universal approaches to thermal design based on direct and inverse problems of combined heat transfer.

The author would like to acknowledge A.A. Alekseev, A.V. Azarov, W.P.P. Fisher, M.L. German, P.S. Grinchuk, S.A. Gusev, O.V. Denisov, A.D. Novikov, K.V. Mikhailovsky, A.M. Mikhalev, L.Ya. Paderin, N.V. Pavlyukevich, I.R. Shafikova and M.S. Tret'yak for their scientific cooperation.

Separate parts of this work are fulfilled with financial support on contract from Russian Ministry of Education and Science on project on Agreement 14.574.21.0146 from 26 September 2017 (Unique identifier of the project RFMEFI57417X0146).

\section{References}

1. S.V. Reznik, P.V. Prosuntsov, A.V. Azarov, J Eng Phys Thermophys. 88, 3 (2015)

2. S.V. Reznik, P.V. Prosuntsov, A.V. Azarov, J Eng Phys Thermophys. 88, 4 (2015)

3. P.V. Prosuntsov, S.V. Reznik, K.V. Mikhailovsky, A.D. Novikov, Zaw Ye Aung, IOP Conf. Series: Materials Science and Engineering 153, (1), 012012 (2016)

4. S.V. Reznik, A.D. Novikov, MATEC Web Conf. 92, 01012 (2017)

5. M.Ya. Gofin, Heat-resistant and thermal protection structures of reusable aerospace vehicles (ZAO "TF MIR”, Moscow, 2003)

6. M.L. Blosser, J Spacecraft Rockets 41, 2 (2004)

7. J.T. Dorsey, C.C. Poteet, K.E. Wurster, R.R. Chen, J Spacecraft Rockets 41, 2 (2004)

8. P.V. Prosuntsov, S.V. Reznik, JOEP 49, 6 (1985)

9. P.V. Prosuntsov, Heat Transfer Research 36, 6 (2005)

10. P.V. Prosuntsov, I.A. Mayorova, A.V. Zuev, Thermal Processes Technology 7 (2014)

11. P.V. Prosuntsov, S.V. Reznik, Siberian Phys.-Tech. J. 1 (1991)

12. P.V. Prosuntsov, S.V. Reznik, V.E. Tyutyunnik, Siberian Phys.-Tech. J. 4 (1992)

13. P.V. Prosuntsov, S.V. Reznik, Siberian Phys.-Tech. J. 4 (1992)

14. S.V. Reznik, P.V. Prosuntsov, A.V. Zuev, Proc. 2-nd Int. Symp. on Inverse Problems, Design and Optimization (Miami, FL, USA, 2017)

15. S.V. Reznik, Abstracts of the Reports and Communications of 5-th Int. Forum on Heat and Mass Transfer (Minsk, Belarus, 2004) 